
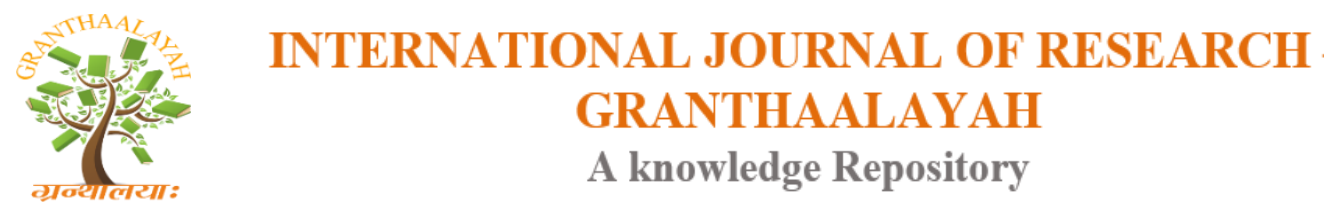

Science

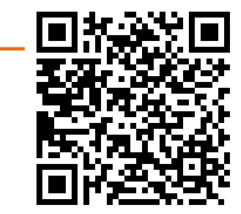

\title{
A BRIEF SURVEY ON UNDERWATER WIRELESS OPTICAL COMMUNICATION SYSTEM
}

\author{
Amayika Kakati ${ }^{1}$, Deeksha Sharma ${ }^{1}$, Nikhil N Aggarwal ${ }^{1}$ \\ ${ }^{1}$ Amity University, Kurkshetra University, Department of Electronics and Communication \\ Engineering, India
}

\begin{abstract}
The underwater wireless optical communication (UWOC) system gives very high rate of underwater data transmission over several meters. It utilizes the visible band in the spectral range of 390-750nm of the electromagnetic spectrum. In this paper, we study various types of link configurations depending upon the circumstances in water environment. We also study the effect of various water types on the system performance. We find that the UWOC system is affected especially by inherent optical properties such as absorption and scattering and the effect of these properties is different in various water types. We also present that misalignment in UOWC system adversely affects its functioning and reliability. Finally, we present that hybrid system design for a proper systematic UOWC system.
\end{abstract}

Keywords: Autonomous Underwater Vehicles (AUV); Link Misalignment; Remotely Operated Underwater Vehicles (ROV); Underwater Wireless Optical Communication (UWOC).

Cite This Article: Amayika Kakati, Deeksha Sharma, and Nikhil N Aggarwal. (2018). "A BRIEF SURVEY ON UNDERWATER WIRELESS OPTICAL COMMUNICATION SYSTEM." International Journal of Research - Granthaalayah, 6(6), 238-245. https://doi.org/10.29121/granthaalayah.v6.i6.2018.1370.

\section{Introduction}

The increase in global warming and reduction of ground has increased the exploration of ocean. The Underwater wireless communication (UWC) technology can achieve the exploration of ocean. The underwater wireless communication is define as the transmission of data in a random water medium with the use of wireless carriers like radio-frequency (RF) waves, acoustic waves, and optical waves [1]. The need of underwater communication technique have increased because of human actions in underwater background like underwater monitoring, exploration, surveillance and off shore oil field exploration. Because of the restricted bandwidth of radio-frequency (RF) and acoustic system and the requirement of high speed underwater data transmission rate underwater wireless optical communication (UWOC) is needed [2]. 
Underwater wireless optical communication (UWOC) is the transmission of data in a random water medium with the use of optical waves. UWOC system give very high rate of underwater data transmission over very large distance [1]. The Underwater wireless optical communication utilizes the visible band in the spectral range of 450-550nm of the electromagnetic spectrum [1]. The Primary Underwater wireless sensor network (UWSN) comprise of a large number of Distributed nodes such as Seabed sensors, relay buoys, autonomous underwater vehicles (AUV) and remotely operated underwater vehicles (ROV).In figure 1,sensors situated in the depth of the seabed gather the data and transmit it via optical link to AUV and ROV. The AUV and ROV transfer the signals to ships, submarines, communication buoys and other underwater vehicles. Above the sea surface, the ground data centre process the data and communicates with satellite and ships through RF or FSO link.

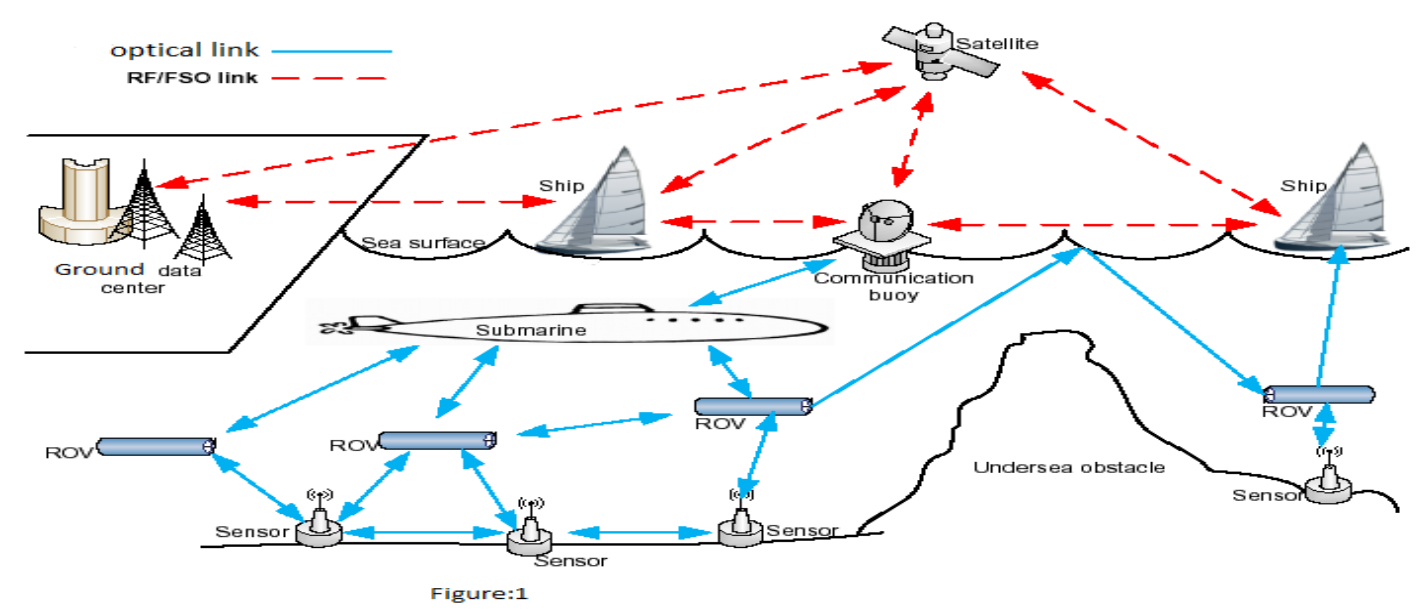

\section{Basic Block Diagram of UWOC System}

The Basic block diagram of underwater optical communication system is shown in figure 2 below. In the transmitter section there is a modulator which modulates the input signal. The optical device can be LASER or LED. The modulated optical signal which contains the information is projected by projection optics and beam steering if necessary into sea water through platform window. At the receiver section the optical signal enters the platform window of receiver after getting attenuated in sea water medium. The received Optical signal after passing through collection optics reaches to detector and then subjected to the signal processing and demodulation process [4].

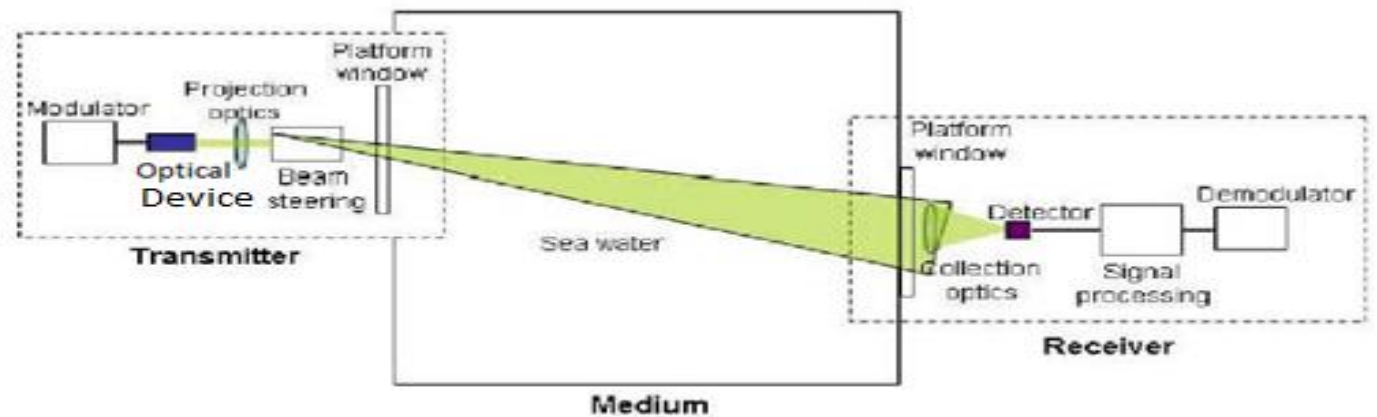

Figure 2 : Block Diagram of a Underwater Optical Communication System(UWOC) 


\section{Link Configuration}

UWOC are divided into four categories based on the link configuration between the nodes in UWSNs as shown in figure 2.

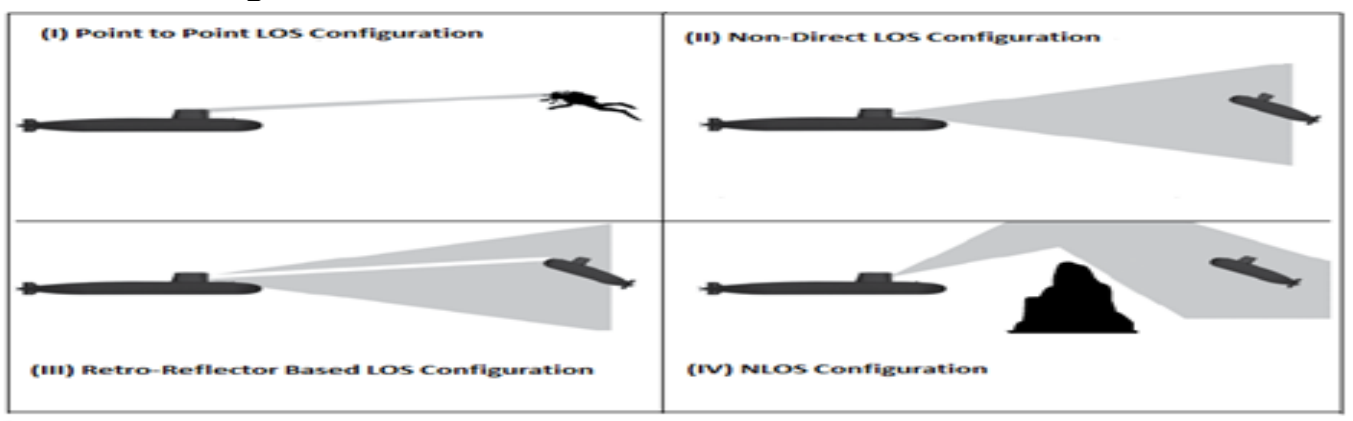

Figure 3 : Different Types of Link Configuration

1) Point to Point LOS configuration is the most usually used link configuration in UWOC. The receiver identifies the optical signal in the direction of the transmitter. Precise pointing is required between transmitter and receiver because the point to point LOS UWOC system have light sources with a narrow divergence angle as in case of a LASER. This type of requirement limit the capability of UWOC system in turbid or turbulent water condition and then become a critical problem when transmitter and receiver are non-stationary nodes like AUV and ROV.

2) Non-Direct LOS configuration have light source with a large divergence angle as in case of high power light emitting diode (LED) to achieve broadcasting UWOC from one node to various nodes. Precise pointing is not required in this configuration link but in comparison with the point to point LOS configuration, the diffused light based link faces aquatic attenuation due to large interaction area with water. Major limitation of this configuration is less communication distance and lower data rates.

3) Retro reflector-based LOS configuration can be considered as a similar realization of point to point LOS configuration. This type of configuration is appropriate for duplex UWOC system which is having limited power and weight budget, for example an underwater sensor node. In modulated retro reflector link, the transmitted signal is reflected back from a modulated retro reflector. In this process the information that the retro reflector reflect to the transceiver will be encoded on the reflected signal. As there is no light source available in the retro reflector side, its power dissipation, volume and weight is magnificently decrease. Limitation of this type of configuration link is that the backscattering of the transmitted optical signal can obstruct the reflected signal, hence it reduce the signal to noise ratio (SNR) and bit error rate (BER). Also, more attenuation occurs when the optical signal travel in underwater channel twice.

4) NLOS configuration solve the alignment problem occurred in LOS UWOC system. In this type of configuration, the transmitter incident the light signal to the sea surface with an angle of incidence more than the critical angle, such that the light signal should undergo a total internal reflection. The receiver must keep facing towards the sea surface in a direction in such a way that it is approximately parallel with the reflected signal to make sure complete signal receiving. The main problem for NLOS link is the non linear sea surface slopes caused by winds or any other disruption source. These unwanted occurrences will reflect light signal back towards the transmitter side and originate intense signal dispersion. 
1) High data transmission rate

2) Low link delay

3) Cost of implementation is low.

4) It has very high signal security as compare to RF and acoustic link

5) More energy efficient and cost effective than RF and acoustic link as small and low cost underwater transceivers are implemented

\section{Disadvantages}

1) Due to the effect of absorption and scattering of optical signal, UWOC system experiences poor BER result over very small distances in turbid water condition.

2) In UWOC system, the optical link sometime gets terminated due to misalignment of optical transceivers.

3) Underwater environment is intricate, due to flow, pressure, temperature and salinity of sea water, the performance and lifetime of optical devices gets effect, so reliable underwater devices are required.

\section{Applications}

1) In real time application like underwater video transmission

2) Environmental monitoring and surveillance

3) Underwater exploration like off shore oil field exploration

\section{Characteristics of Underwater Optical Channel}

\section{1) Light Propagation in Water}

The optical property of water is categories as: inherent optical property (IOP) and apparent optical property (AOP).

\section{- Inherent Optical Property (IOP)}

The Inherent optical property (IOP) is an optical parameter which only relies on the transmission channel. They are not dependent on the characteristic of optical source. In UWOC system, IOP is used in calculation link budgets and it has extensive effect on link performance. The IOP of water are absorption, scattering, attenuation and volume scattering function. Absorption and scattering are wavelength dependent. Absorption and scattering are the two which mainly affect the propagation of light in water [1] [3].

>>Absorption is unchangeable dissipation of light intensity and it is dependent on the refractive index of water. The effect of absorption in a UWOC system is the overall propagation energy of optical signal gets decreases which can restrict the link distance [3]. The absorption coefficient is $\mathrm{a}(\lambda)$.

>>While scattering occurs when light gets deflect from its actual path. The effect of scattering in a UWOC system is as the size of optical aperture is fixed, the scattering will spread the optical signal and as a result the number of photon reach at the receiver side get reduces. This causes degrading of Signal to noise ratio (SNR) in the UWOC system. Second, due to scattering of optical 
signal in UWOC system, the photons may reach at the receiver side at separate time slots each and results in multi-path dispersions like Inter symbol interference (ISI) and Timing jitter [1][3]. The scattering coefficient is $b(\lambda)$.

The total attenuation caused by absorption and scattering is defined by attenuation coefficient $\mathrm{c}(\lambda)$, which is $c(\lambda)=a(\lambda)+b(\lambda)$. The unit of absorption, scattering and attenuation coefficient is $\mathrm{m}^{-1}$

>> There are two types of attenuation coefficient as shown in figure 4. First is beam attenuation coefficient, which is used for calculating attenuation of accurately parallel beam like LASER [4]. It is denoted by c. Second is diffuse attenuation coefficient, which is used for calculating attenuation of diffuse light ray like sunlight, as sunlight travel from ocean surface to depth in water [4]. It is denoted by K. They are defined as:

For Beam attenuation coefficient, $\mathrm{I}_{\mathrm{t}}=\mathrm{I}_{0} \exp (-\mathrm{cz})$ and For Diffuse attenuation coefficient, $\mathrm{I}_{\mathrm{t}}=\mathrm{I}_{0}$ $\exp (-\mathrm{Kz})$. Where, $\mathrm{I}_{t}=$ transmitted irradiance, $\mathrm{I}_{0}=$ Initial light radiance and $\mathrm{z}=$ distance travel by light [4]

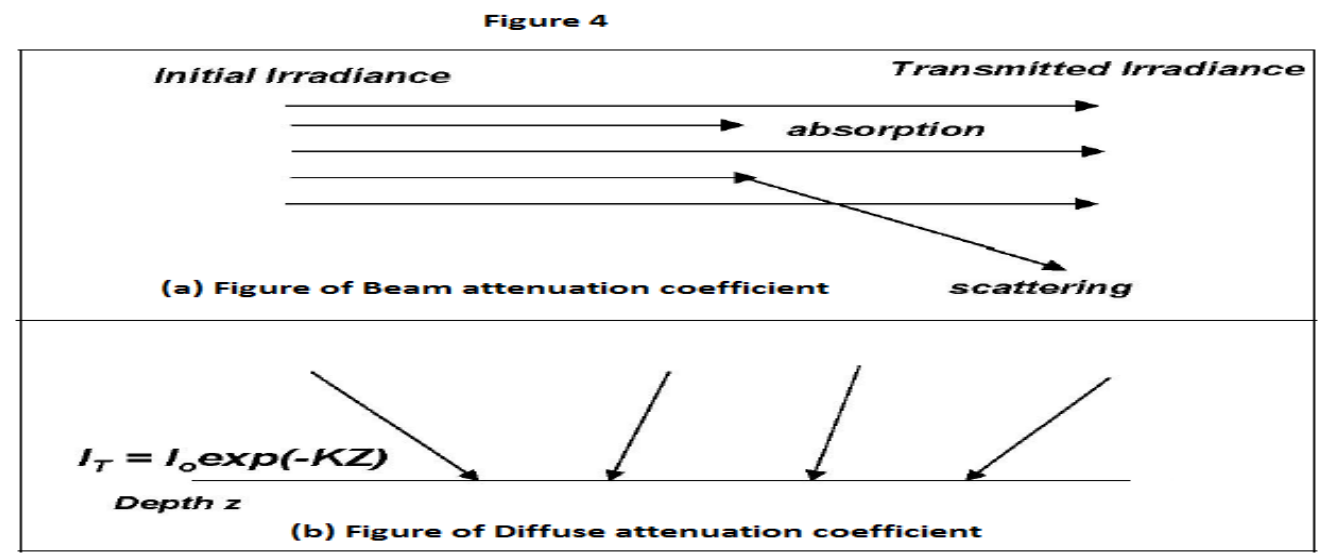

> The volume scattering function (VSF) is describe as the angle $\psi$ obtained by scattered light when the Incident light comes in contact with a particle in water [3] as shown in figure 5. It is denoted by $\beta(\psi, \lambda)$. The VSF can provide the Inherent Optical property(IOP) of a medium[3]. Scattering coefficient can be obtained by integrating the VSF in every direction [3]. It is describe as:

$b(\lambda)=2 \pi \int_{0}^{\pi} \beta(\psi, \lambda) \sin (\psi) d \psi$

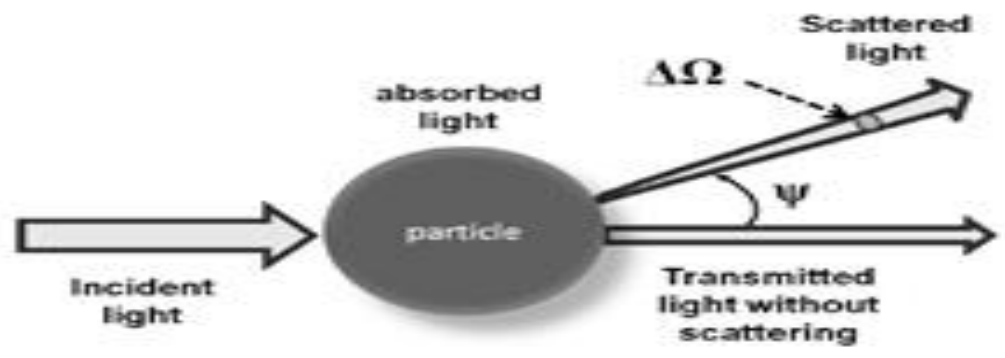

Flgure S: Light scatrering when comes in contact with a particle in water [2] 


\section{- Apparent Optical Property (AOP)}

The apparent optical property (AOP) is an optical parameter which relies not only in the transmission channel but also on the geometrical structure of the optical field. The AOP of water are radiance, irradiance and reflectance. Also due to turbulence in ocean, the functioning of the UWOC system gets effected due to the occurrence of fading channel. Turbulence in water is caused due to change in the refractive index of water that is because of the variation in temperature, pressure and salinity of water.

\section{2) Types of Water}

1) There are four significant kinds of water types. The standard of water is different from one area to another [3].

2) Pure sea water: In pure sea water absorption is the main light limiting fact. The small value of $b$ and angle scattering in direction of travelling can create the light to travel uniformly in one direction only.

3) Clear ocean water: In Clear Ocean water, there is intense mass of diffuse particles that influence scattering.

4) Coastal ocean water: In coastal ocean water, there is large amount of intense mass of plank tonic matters, detritus and mineral part that influence scattering and absorption.

5) Turbid harbor and estuary water: This water type has extreme intense mass of diffuse and interruption substances.

\section{3) Link Misalignment in UWOC}

Misalignment in any communication link can severely affect its functioning and reliability. It generally occurs in small field of view (FOV) receivers. Link misalignment in UWOC can occur due to various reasons, first is Limitation of transceivers such that in some applications to obtain high data rate and long-distance communication range LASER diode is used in transmitting and Photo diode is used at the receiver end. LASER diode has small divergence angle and finite FOV of Photo diode causes misalignment. This misalignment can be overcome by using array of LEDs in transmitting side and array of Photo diodes in the receiver end such that it provides large FOV receiver [1-2]. Secondly UWOC link misalignment occur when connecting with underwater vehicles like AUV, ROV etc as they are continuously moving in water. These underwater vehicles must be tracked by the transceivers. Third is because of flow of ocean and wind can cause movement of transceivers in UWOC system and last due to change in refractive index of water in depth, salinity and temperature, it result in non linear propagation of optical signal. This type of misalignment occurs in surface to bottom UWOC link.

\section{Hybrid UWOC System}

In Hybrid UWOC System two type of hybrid link have been design. The first hybrid link design makes use of optical and acoustic waves together as medium in duplex communication as shown in figure 6.A.In this design the link is build up between the underwater vehicles. All the underwater vehicles are outfitted with optical and acoustic transceivers together. If the distance between the underwater vehicles is small and the water is free from any obstruction, the system uses optical wave which gives extremely high rate of transmission of data and if the distance between the 
underwater vehicles is large and the water is turbid, the system will make use of acoustic wave to achieve transmission. The advantage of this hybrid link design is openness to change according to distance and condition of water. Also it is reliable. The disadvantage is high power utilization and heavily built acoustic transceivers in the underwater vehicles [1].

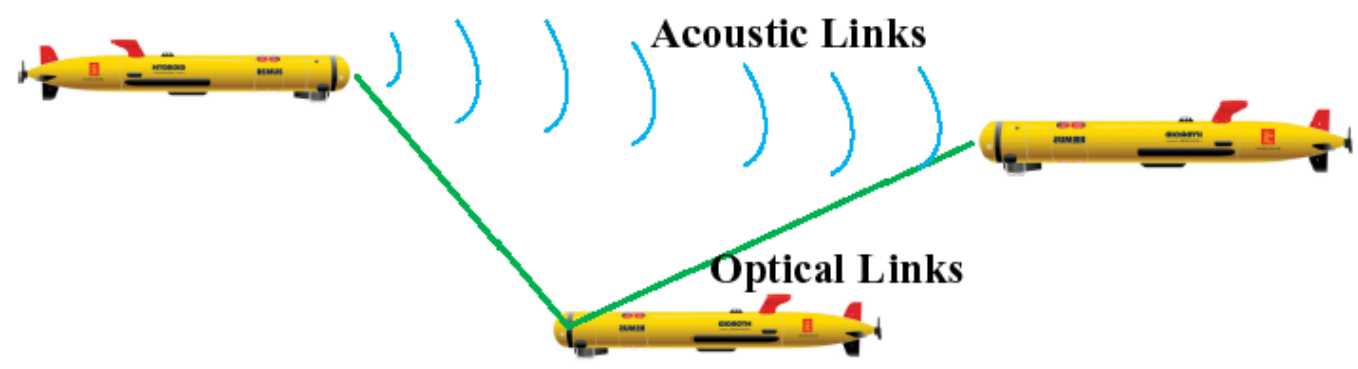

Figure 6.A :Mutual UWOC Hybrid link design

The second Hybrid link design as shown in figure 6.B contains one stable control platform and various underwater sensor vehicles. Data from control platform is transmitted to each underwater sensor vehicles in downlink by acoustic waves and the information between the underwater sensor vehicles is carried out by optical waves. The optical waves also carry the information from underwater sensor vehicles to the control platform in uplink [1]. In this UWOC Hybrid design the acoustic waves have diffusion and large distance propagation property, the acoustic wave is extended over the area formed by the underwater vehicles [1]. Furthermore the control signals from control platform to the underwater vehicles in downlink carry low data rate, which is appropriate for acoustic communication. While the large volume of underwater monitored information is transmitted by high speed optical waves.

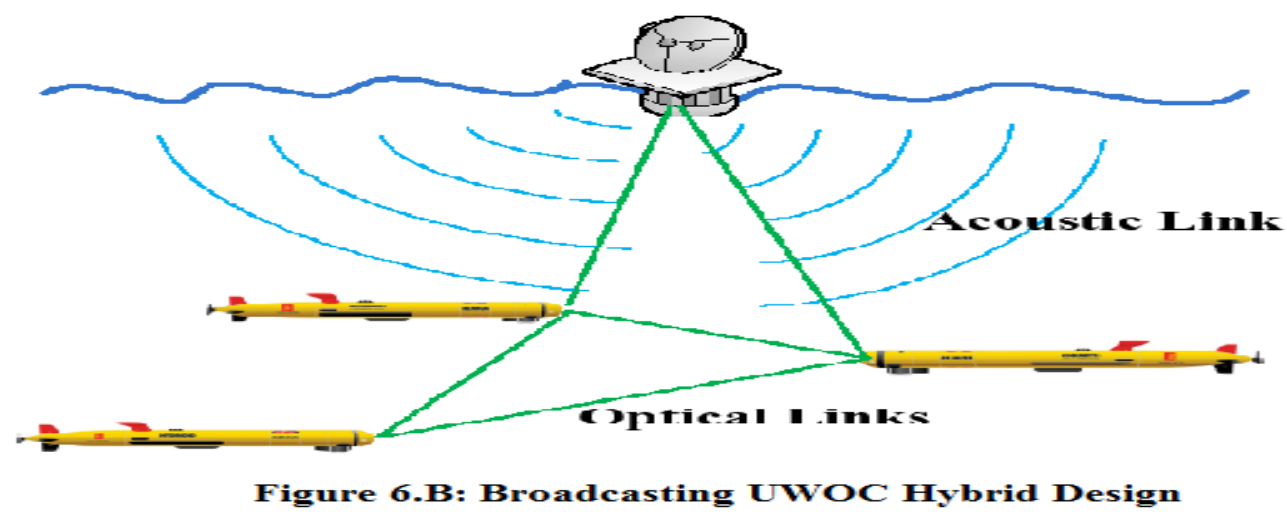

\section{References}

[1] A THESIS on, "A Survey of Underwater Wireless Optical Communication", by Zhaoquan Zeng, submitted to THE COLLEGE OF GRADUATE STUDIES (Electrical Engineering), THE UNIVERSITY OF BRITISH COLUMBIA(Okanagan), December 2015

[2] Mohammad-Ali Khalighi, Chadi Gabriel, Tasnim Hamza, Salah Bourennane, Pierre León, Vincent Regaud, "Underwater Wireless Optical Communication; Recent Advances and Remaining Challenges,” IEEE Transparent Optical Networks (ICTON), pp. 1-4,6-10 July 2014 
[3] Chadi Gabriel, Mohammad-Ali Khalighi, Salah Bourennane, Pierre León, "Channel Modeling for Underwater Optical Communication”, IEEE GLOBECOM Workshops (GC Wkshps), pp. 833837,5-9 Dec. 2011

[4] John W. Giles and Isaac N. Bankman, "Underwater Optical Communications Systems Part: 2 Basic Design Considerations," IEEE Military Communications Conference, 2005. MILCOM 2005, Vol .3, pp. 1700-1705, 17-20 Oct. 2009

*Corresponding author.

E-mail address: Amy24sc@ gmail.com, deekshas1820@ gmail.com, nikhilaggarwal296@ gmail.com 